The connection between oscillations and feedback in cellular regulatory systems goes back to the 1960s and early 1970s, when experimentalists studying glycolytic oscillations, mitotic and circadian rhythms, periodic enzyme synthesis and cyclic AMP oscillations joined forces with theoreticians to speculate on underlying molecular mechanisms that were, in many cases, beyond the power of the biochemical techniques of the day ${ }^{9}$. In the 1980s' frenzy to crack open these black boxes with the tools of molecular genetics, the rapport between experimentalists and theoreticians fell by the wayside. The work of Lahav et al. joins several recent publications that demonstrate a renewed vitality of molecular genetics when combined with sound theoretical and computational analysis of macromolecular regulatory systems $^{10-14}$. This rapprochement promises deeper insights into how a cell tailors its response to internal and external signals.

\footnotetext{
1. Hanahan, D. \& Weinberg, R.A. Cell 100, 57-70 (2000).

2. Lahav, G. et al. Nat. Genet. 36, 147-150 (2004).

3. Bray, D. Nature 376, 307-312 (1995).

4. Ferrell, J.E. Jr \& Xiong, W. Chaos 11, 227-236 (2001).

5. Tyson, J.J., Chen, K.C. \& Novak, B. Curr. Opin. Cell Biol. 15, 221-231 (2003).

6. Lev Bar-Or, R. et al. Proc. Natl. Acad. Sci. USA $\mathbf{9 7}$,
}

11250-11255 (2000)

7. Dayan, P. \& Abbott, L.F. Theoretical Neuroscience: Computational and Mathematical Modeling of Neural Systems (MIT Press, Cambridge, 2001).

8. Goldbeter, A. Biochemical Oscillations and Cellular Rhythms (Cambridge University Press, Cambridge, 1996).

9. Chance, B., Pye, E.K., Ghosh, A.K. \& Hess, B. (eds.) Biological and Biochemical Oscillators (Academic, New York, 1973).

10. Elowitz, M.B. \& Leibler, S. Nature 403, 335-338 (2000).

11. Hoffman, A., Levchenko, A., Scott, M.L. \& Baltimore, D. Science 298, 1241-1245 (2002).

12. Xiong, W. \& Ferrell, J.E. Jr Nature 426, 460-465 (2003).

13. Sha, W. et al. Proc. Natl. Acad. Sci. USA 100 975-980 (2003)

14. Cross, F. Dev. Cell 4, 741-752 (2003).

\title{
Close look at gene conversion hot spots
}

\author{
Jeffrey D Wall
}

\begin{abstract}
New work has directly estimated male gene conversion rates in three regions of the human genome and identified gene conversion hot spots in the same locations as previously identified crossover hot spots. This work elucidates the fine-scale structure of linkage disequilibrium (LD) in the human genome and will be useful in association studies and other LD-based applications in population and human genetics.
\end{abstract}

Standard models suggest that there are two different forms of homologous recombination, commonly called crossover (which may be accompanied by gene conversion) and gene conversion (without an associated crossover $)^{1}$. Crossover rates vary tremendously across the human genome ${ }^{2}$, by several orders of magnitude over distances as small as $1 \mathrm{~kb}$ (ref. 3). But there are few direct data on homologous gene conversion rates ${ }^{4}$, and no information on the extent to which gene conversion rates vary across the genome. In the accompanying paper, Alec Jeffreys and Celia May ${ }^{5}$ estimated gene conversion rates at three known crossover hot spots in humans by sperm typing. They found that all three regions were gene conversion hot spots as well and that in each case, the location of the peak of conversion activity coincided with the peak of crossover rates. The coincidence of these peaks suggests that the molecular mechanisms generating most crossovers and gene conversion events are related.

\section{Recombination and LD}

Recombination is one of the primary factors that affect LD (the nonrandom association of alleles at different sites). Standard popu-

Jeffrey D. Wall is in the Program in Molecular and Computational Biology at The University of Southern California, 1042 W. 36th Place, DRB 289, Los Angeles, California 90089, USA.

e-mail: jeffwall@usc.edu lation genetics models of recombination generally ignore gene conversion, even though crossovers and gene conversions have different effects on the structure of LD. Recombination between pairs of markers that are far apart are almost exclusively crossovers, whereas pairs of markers that are close together are affected by both crossovers and gene conversion events. This is crucial for interpreting patterns of

human sequence variation. For example, analyses of human data have found less LD than expected over short distances (e.g., $<5 \mathrm{~kb}$ ) given the $\mathrm{LD}$ observed over longer distances (e.g., >100 kb; ref. 6). This seemingly discordant observation is exactly what would be expected under a model of recombination that incorporates both crossovers and gene conversion. In addition, adding gene conversion to

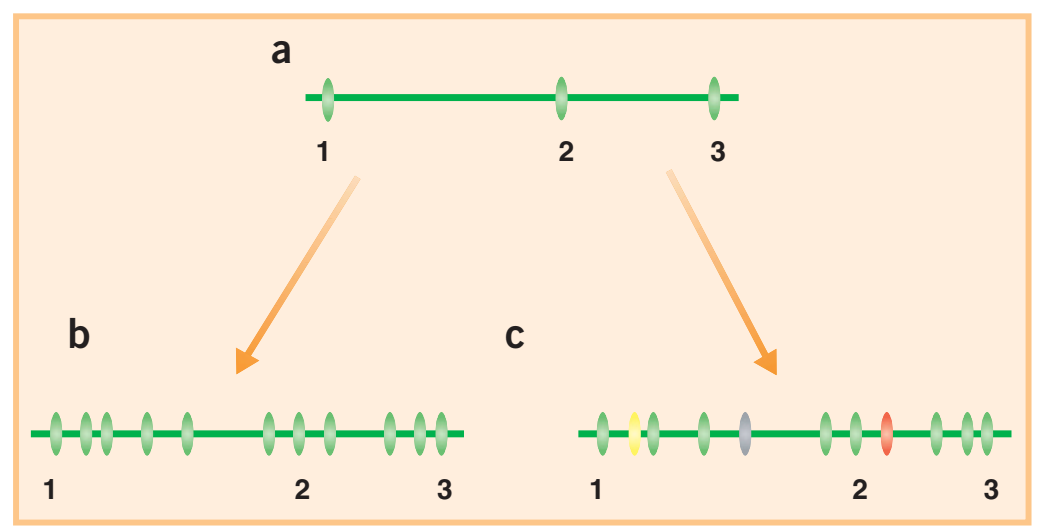

Figure 1 Schematic of the possible effects of gene conversion and marker density on patterns of LD. Horizontal lines represent chromosomes, and ovals represent markers. Ovals that are the same color are in strong LD with each other. (a) Three typed markers (labeled 1, 2 and 3) are in strong LD with each other. The patterns of LD of all the unobserved markers between these three are unknown. (b) One possibility, assumed in much of the discussion of haplotype blocks, is that all the unobserved markers are in strong LD with the end markers. (c) Another possibility, which is more probable if there are high rates of gene conversion or the original markers were far apart, is that many of the unobserved markers are in strong LD with the original three but some are not. Whether real data look more like b or $\mathbf{c}$ depends on both experimental parameters (e.g., sample size, marker density) and intrinsic parameters (e.g., gene conversion rate, crossover rate). 
our models will make other population genetics applications, such as inference of population history from patterns of $\mathrm{LD}$, both easier and more reliable.

The effect of gene conversion on LD also affects human genetics, a primary goal of which is to identify the genetic variants that affect susceptibility to complex diseases. Much recent work on the subject focuses on association mapping methods that use LD. Association mapping attempts to identify causal variants by typing many (e.g., thousands) single-nucleotide polymorphisms (SNPs) in a sample of unrelated individuals and then determining whether any of the SNPs are associated with the disease phenotype of interest. The rationale is that even if the SNPs that were typed do not directly affect disease susceptibility, they will be in strong LD with markers that do. The optimal marker density and success of association studies depend on the fine-scale structure of LD, and in particular on the expected decay of LD with physical distance. Jeffreys and May ${ }^{5}$ estimated that most recombination events are gene conversions $(\sim 80-94 \%$ of events) and that mean tract lengths (i.e., size of the converted piece) are small ( 55-290 bp). For these parameter values, gene conversion would more than double the effective recombination rate between closely spaced markers (e.g., ones within $2 \mathrm{~kb}$ of each other) but would have little effect on pairs of distant markers.

This might be important when trying to predict levels of LD between typed and untyped markers. Generally, when two nearby markers are in strong LD with each other, it is assumed that all markers in between are in strong LD with both end markers. This is the motivation for several recent definitions of 'haplotype blocks' (reviewed in ref. 7). But when the gene conversion rate is high and the marker density is low, it is possible that the intervening SNPs are not in strong LD with the end markers ${ }^{7}$ (Fig. 1). These SNPs can be thought of as 'holes' in haplotype blocks; these holes would reduce the efficacy of association studies. Further empirical and theoretical studies are needed to determine what practical effect, if any, this will have on future association studies.

\section{Recombination rate variation}

A more detailed assessment of the potential relevance of gene conversion to patterns of $\mathrm{LD}$ and to questions in population and human genetics would require data on gene conversion rates and conversion tract lengths from many other regions of the genome. Because estimating gene conversion parameters indirectly from patterns of LD is rather difficult to do accurately ${ }^{8}$, more direct experimental data, for example, from sperm typing, would be a welcome development.

Jeffreys and May ${ }^{5}$ chose to study known crossover hot spots in the MHC in part because the high recombination rates and high marker densities made direct parameter estimation much easier. But because the MHC hot spot regions may be atypical, it is not clear whether the gene conversion parameter estimates from these regions are applicable to the rest of the genome. The fraction of recombination events that are gene conversions may be higher in regions of low crossover rate, and mean conversion tract lengths may vary with levels of heterozygosity ${ }^{9-11}$. These empirical questions will eventually be answered. But comparable sperm typing studies in regions of the genome with average recombination rates (e.g., with crossover rates of $\sim 1.3 \mathrm{cM}$ per $\mathrm{Mb}$; ref. 12) will have to screen many more sperm to recover the same number of recombinants and, because of the lower marker densities, will be less informative about the distribution of conversion tract lengths.

For now, the results of Jeffreys and $\mathrm{May}^{5}$, together with recent work from the Jeffreys laboratory (and other laboratories), raise a host of other questions about recombination rate variation. What is the scale over which crossover and gene conversion rates vary across the human genome? How common are recombination hot spots? Are there regions that are hot spots for either crossover or gene conversion activity but not both? How strongly are male recombination rates, which can be estimated by sperm typing, correlated with female recombination rates? Given that there can be large differences in recombination rates across individuals ${ }^{2,13}$, there is also the possibility that there are large differences in recombination rates between different human populations.

1. Szostak, J.W. et al. Cel/ 33, 25-35 (1983).

2. Arnheim, N., Calabrese, P. \& Nordborg, M. Am. J. Hum. Genet. 73, 5-16 (2003).

3. Jeffreys, A.J. et al. Nat. Genet. 29, 217-222 (2001).

4. Zangenberg, G. et al. Nat. Genet. 10, 407-414 (1995).

5. Jeffreys, A. \& May, C. Nat. Genet. 36, 151-156 (2004).

6. Pritchard, J. \& Przeworski, M. Am. J. Hum. Genet. 69, 1-14 (2001).

7. Wall, J. \& Pritchard, J. Nat. Rev. Genet. 4, 587-597 (2003).

8. Ptak, S., Voelpel, K. \& Przeworski, M. Genetics (in the press).

9. Borts, R. \& Haber, J.E. Science 237, 1459-1465 (1987).

10. Langley, C. et al. Genetics 156, 1837-1852 (2000).

11. Andolfatto, P. \& Wall, J. Genetics 165, 1289-1305 (2003).

12. Kong, A. et al. Nat. Genet. 31, 241-247 (2002).

13. Jeffreys, A. \& Neumann, R. Nat. Genet. 31, 267-271 (2002)

\title{
Getting our AKT together in schizophrenia?
}

\author{
Joachim Hallmayer
}

\begin{abstract}
AKT1 is a protein kinase that functions as a central element in many pathways involved in the control of cell growth and apoptosis. Genetic and biochemical data now provide evidence that $A K T 1$ may be a susceptibility gene for schizophrenia.
\end{abstract}

Since Bleuler ${ }^{1}$ first introduced the term in 1911, schizophrenia has been the subject of controversy. The concept of schizophrenia

Joachim Hallmayer is in the Department of Psychiatry and Behavioral Sciences, Stanford University School of Medicine, Palo Alto, California 94304, USA. e-mail: joachimh@stanford.edu has changed substantially over time, and its boundaries in relation to other types of psychosis (bipolar disorder, for example) are far from clear. Discussion of the etiology of schizophrenia has shifted away from the mother-infant relationship to brain imaging and molecular genetics. But one finding has been replicated repeated: schizophrenia is familial, occurring at a higher frequency among relatives of individuals with the disorder. Coupled with the fact that the concordance rate is much higher in monozygotic twins than in dizygotic twins, this is compelling evidence of a genetic causation. With the advent of molecular approaches, identification of genes associated with schizophrenia has become the 'holy grail' in the field, and on page 131 of this issue, Effat Emamian and 